\title{
As Relações de Otimismo e Bem-Estar Subjetivo entre Pais e Filhos
}

\author{
Cláudia de Moraes Bandeira - UNIL AS ALLE, Canoas, Brasil \\ Jean Carlos Natividade - Universidade Católica do Rio de Janeiro, Rio de Janeiro, Brasil \\ Claudia Hofheinz Giacomoni - Universidade Federal do Rio Grande do Sul, Porto Alegre, Brasil
}

\begin{abstract}
Resumo
Os objetivos deste estudo foram verificar a relação entre o otimismo e o bem-estar subjetivo de pais e filhos e investigar os níveis de otimismo e bem-estar das crianças e dos adultos. Participaram 390 crianças entre quatro e 11 anos e 287 pais, com idades entre 24 e 77 anos. Os meninos apresentaram média significativamente mais alta que as meninas na subescala de pessimismo, e as mães apresentaram médias significativamente superiores aos pais na satisfação de vida. A satisfação de vida dos pais apresentou correlação com a satisfação de vida das mães. Os resultados indicaram correlações positivas entre o otimismo dos filhos e os afetos positivos das mães e entre a satisfação de vida dos filhos e o otimismo das mães. Apenas variáveis maternas apresentaram relação com indicadores de um desenvolvimento positivo.
\end{abstract}

Palavras-chave: Otimismo, bem-estar subjetivo, pais-filhos.

\section{Correlations of Optimism and Subjective Well-Being between Parents and Children}

\begin{abstract}
The aims of this study were to verify the relation of the subjective well-being of parents and their children, and to present optimism and well-being levels in groups of adults and children. Participants were 390 children aged between four and 11 years of age and 287 parents, aged between 24 and 77. Boys scored higher than girls in pessimism, and mothers showed more satisfaction with life than fathers. Fathers' life satisfaction was correlated with mothers' life satisfaction. Results showed mother's optimism was positively correlated with children's life satisfaction, and mothers' positive affect correlated positively with children's optimism. Only the variables involving mothers showed a relationship with indicators of a positive development.

Keywords: Optimism, subjective well-being, parent-child.
\end{abstract}

Las Relaciones del Optimismo y el Bienestar Subjetivo entre Padres e Hijos

\begin{abstract}
Resumen
Los objetivos de este estudio fueron verificar la relación entre el optimismo y el bienestar subjetivo de padres e hijos, e investigar los niveles de optimismo y bienestar subjetivo de niños y adultos. Participaron 390 niños de edades entre 4 y 11 años y 287 padres, con edades entre 24 y 77 años. Los niños presentaron media significativamente más alta que las niñas en la sub-escala de pesimismo, y las madres presentaron medias significativamente superiores a los padres en la satisfacción con la vida. La satisfacción con la vida de los padres mostró correlación con la satisfacción de vida de las madres. Los resultados mostraron una correlación positiva entre el optimismo de los niños y los afectos positivos de las madres, y entre la satisfacción vital de los hijos y el optimismo de las madres. Sólo variables maternas se relacionaron con los indicadores de desarrollo positivo.

Palabras clave: Optimismo, bienestar subjetivo, padres e hijos.
\end{abstract}

\section{Introdução}

Sem negar a doença, as dificuldades e os problemas, a perspectiva positiva na psicologia busca estudar, entender e valorizar as forças e as potencialidades humanas. A Psicologia Positiva reúne pesquisadores que estudam as experiências positivas subjetivas, os traços positivos das pessoas e as instituições que permitem as experiências e manifestação desses traços (Seligman \& Csikszentmihalyi, 2000). Esse movimento dentro da Psicologia adota uma postura mais apreciativa dos potenciais, motivações, e capacidades dos indivíduos. A Psicologia Positiva procura transformar antigas questões em novas oportunidades de entender eventos psicológicos, como, por exemplo, o otimismo, a criatividade, o altruísmo e o bem-estar, considerando-os tão importantes para a investigação científica como a ansiedade, a depressão e a agressividade (Roberts, Brown, Johnson, \& Reinke, 2005; Seligman \& Csikszentmihalyi, 2000). As pesquisas, nesse campo, visam a identificar fatores que melhoram o desenvolvimento, o bem-estar e as capacidades individuais (Chang, 2002; Diener, 2000; Ey et al., 2005; Larson, 2006; Roberts et al. 2005; Scheier \& Carver, 1985).

Um dos temas estudados pela Psicologia Positiva é o desenvolvimento positivo, uma nova abordagem que visa promover o desenvolvimento saudável das crianças e de suas famílias (Lerner, Almerigi, Theokas, \& Lerner, 2005). Compreende-se desenvolvimento positivo como os aspectos positivos do 
comportamento e os resultados positivos do processo desenvolvimental. Para essa abordagem, a ênfase é dada na plasticidade do desenvolvimento e na importância das relações entre o indivíduo e suas configurações ecológicas. As crianças são vistas a partir das suas potencialidades a serem desenvolvidas (Larson, 2006; Lerner et al., 2005).

Vários estudos têm apontado para a importância de uma perspectiva otimista (e.g. Chang, 2002; Ey et al., 2005; Roberts et al., 2005; Scheier \& Carver, 1985). Indivíduos otimistas e resilientes, por exemplo, apresentam menor probabilidade de desenvolver depressão e ansiedade e é muito mais provável que desempenhem bem na escola, no trabalho, na política e nos esportes (Carver, Scheier, \& Segerstrom, 2010; Ey tet al., 2005; Scheier \& Carver, 1985).

O otimismo pode auxiliar no aperfeiçoamento dos recursos cognitivos, contextuais e de enfrentamento que promovem uma melhor saúde mental (Carver et al., 2010). O otimismo também está associado positivamente ao bem-estar psicológico e físico e à satisfação de vida em adultos, e está inversamente relacionado com desesperança, um fator de risco para doenças depressivas (Carver et al., 2010; Chang, 2002; Scheier \& Carver, 1985). Crianças e adolescentes mais otimistas quanto às suas habilidades para superar dificuldades demonstram mais autovalor, competência e menos sintomas depressivos (Ey et al., 2005).

Conceitualmente, otimismo e pessimismo são expectativas gerais e difusas em relação ao futuro (Carver et al., 2010; Scheier \& Carver, 1985). Na teoria do Otimismo Disposicional (Scheier \& Carver, 1985), os otimistas são as pessoas que: (a) esperam que coisas boas aconteçam, (b) percebem os resultados esperados como atingíveis e (c) persistem na direção de seus esforços. Em contraste, os indivíduos que apresentam expectativas negativas para o futuro estão mais propensos a esperar que coisas ruins aconteçam em suas vidas, esforçam-se menos, tornam-se passivos e desistem mais facilmente de seus objetivos (Carver et al., 2010; Scheier $\&$ Carver, 1985).

Por sua vez, o bem-estar subjetivo (BES) é estudado como um construto multidimensional constituído por componentes afetivos e cognitivos (Diener, 2000; Diener, Oishi, \& Lucas, 2003). O componente afetivo envolve experiências de emoções prazerosas (afeto positivo) e experiências de emoções negativas (afeto negativo) e o componente cognitivo envolve o julgamento individual em relação à qualidade da vida (Diener et al., 2003).
O contexto no qual as crianças se desenvolvem influenciam seus níveis de otimismo e bem-estar subjetivo (Desjardins, Zelenski, \& Coplan, 2008). Apesar do aumento na quantidade de estudos direcionados ao otimismo e ao BES (e.g. Carver et al., 2010; Desjardins et al., 2008; Diener, 2000; Diener et al., 2003; Scheier \& Carver, 1985), ainda são poucos os estudos que investigam a relação dessas variáveis entre pais e filhos.

É possível que os níveis de otimismo e de BES dos filhos sejam influenciados pelos níveis de otimismo e de BES de seus pais, em parte pela modelagem e aprendizagem social, e em parte pelos fatores hereditários. Scheier e Carver (1985) acreditam que as crianças podem adquirir o senso de otimismo e pessimismo de seus pais. Assim, se o otimismo e o BES dos filhos são, de alguma forma, influenciados pelo otimismo e o BES de seus pais, seriam esperadas correlações significativas nos escores de otimismo e BES de pais e filhos. Entretanto, os resultados de estudos que buscam tais correlações se mostram inconsistentes (e.g. Desjardins et al., 2008; Hasan \& Power, 2002; Heinonen, Räikkönen, \& Keltikangas-Järvinen, 2005; Tomasi, 2013).

Os resultados de um estudo que buscou correlações entre otimismo e pessimismo de mães e filhos indicaram que o pessimismo das mães correlacionou-se positivamente com o pessimismo dos filhos, enquanto o otimismo não apresentou correlação significativa (Hasan \& Power, 2002). Esses achados se assemelham aos do estudo de Heinonen, Räikkönen e Keltikangas-Järvinen (2005) que também não encontraram relações entre o otimismo de pais e filhos. Na mesma linha, Tomasi (2013) investigou as relações entre bem-estar de pais e filhos adolescentes, e os resultados apontaram correlações baixas entre essas variáveis. Os resultados desse estudo destacaram a importância da satisfação de vida dos pais para o bem-estar dos filhos, porém o bem-estar dos pais não se mostrou influenciado pela satisfação de vida dos filhos. Outro estudo evidenciou correlação entre a satisfação de vida materna com a diminuição nos problemas de comportamento dos filhos e o aumento do desempenho cognitivo das crianças (Berger \& Spiess, 2011).

A presença de pais cuidadosos, de segurança financeira e de estilos parentais autoritativos funciona como preditores de otimismo na idade adulta (Ey et al., 2005; Heinonen et al., 2005). Um estudo brasileiro buscou investigar a relação entre o otimismo da criança e os estilos parentais (Weber, Brandenburg, \& Viezzer, 2003). Os resultados demonstraram que pais autoritativos foram associados a maiores escores de 
otimismo, enquanto pais negligentes foram associados a menores escores de otimismo. O relacionamento entre pais e filhos.

Embora a literatura aponte para a importância dos adultos no desenvolvimento positivo das crianças (Larson, 2006; Lerner et al., 2005), a forma como os pais influenciam o otimismo e o BES dos filhos ainda não está claro. Os adultos desempenham um papel fundamental ao auxiliar as crianças e os adolescentes a mobilizar o seu potencial para o crescimento (Larson, 2006). Tomando por base o pressuposto de que tanto os aspectos pessoais quanto os do contexto têm sido apontados como fundamentais na compreensão do otimismo e do BES ao longo do desenvolvimento. Este estudo teve como objetivo verificar as relações de otimismo e BES entre pais e filhos.

\section{Método}

\section{Participantes}

Participaram deste estudo 390 crianças entre quatro e 11 anos $(M=7,98 ; D P=2,16)$, estudantes do ensino básico e fundamental de duas escolas da região sul do Brasil, sendo que 53\% eram meninas. Também participaram 287 responsáveis pelas crianças, sendo $56,8 \%$ mães com idades entre 24 e 65 anos $(M=39,6$; $D P=6,49)$ e $43,2 \%$ pais com idades entre 26 e 77 anos $(M=43,6 ; D P=8,16)$.

\section{Instrumentos}

Os instrumentos utilizados para avaliar o otimismo e BES das crianças são descritos a seguir. Youth Life Orientation test - YLOT (Bandeira, Giacomoni, \& Hutz, 2014a). Essa escala afere o otimismo a partir de dois fatores, otimismo e pessimismo. A consistência interna geral da escala, coeficiente alfa, foi adequada $(\alpha$ $=0,81)$, bem como nas subescalas de otimismo $(\alpha=$ $0,74)$ e pessimismo $(\alpha=0,73)$. A YLOT é composta por 12 itens, seis para o otimismo e seis para o pessimismo, e utiliza uma escala de respostas do tipo Likert de quatro pontos. Essa escala foi desenvolvida originalmente para crianças entre oito e dezesseis anos. Para a realização deste estudo, a escala foi adaptada para a utilização em crianças menores de oito anos. Os itens foram lidos e, para auxiliar nas respostas, foi criada uma escala pictórica de quatro pontos onde as crianças apontavam o quadrado correspondente à resposta escolhida: (a) concordo totalmente (quadrado completamente cheio), (b) concordo (quadrado parcialmente cheio), (c) discordo (quadrado parcialmente vazio) e (d) discordo totalmente (quadrado totalmente vazio).

Escala de Afetos Positivo e Negativo para Crianças (Giacomoni \& Hutz, 2006). Essa escala é composta por 30 itens, sendo 15 itens da subescala de afeto positivo e 15 itens da subescala de Afeto Negativo. É utilizada uma escala de respostas Likert de cinco pontos, que variam de um (nem um pouco) a cinco (muitíssimo). O coeficiente alfa da escala total, nos estudos de construção da escala foi de $\alpha=0,90$. Para a subescala de Afeto Positivo, o índice foi $\alpha=0,88$ e, para a subescala de Afeto Negativo, foi de $\alpha=0,84$. Assim como para a YLOT, essa escala foi adaptada para a utilização nas crianças menores de oito anos. A aplicação foi individual e os itens foram lidos para a criança. Para auxiliar nas respostas, foi criada uma escala pictórica de cinco pontos onde as crianças apontavam o quadrado correspondente à resposta escolhida: (a) muitíssimo (quadrado completamente cheio), (b) bastante (quadrado quase cheio), (c) mais ou menos (quadrado pela metade), (d) um pouco (quadrado um pouco cheio), (e) nem um pouco (quadrado vazio).

Escala Multidimensional Satisfação de Vida para Crianças - versão reduzida (Giacomoni, Bandeira, \& Zanon, 2014). A escala Multidimensional de Satisfação de Vida para Crianças é uma medida cujo objetivo é avaliar a satisfação de vida das crianças a partir de domínios específicos de suas vidas. Em sua versão reduzida, a escala possui 27 itens divididos entre quatro fatores: (a) self comparado, (b) família, (c) amizade e (d) escola. O estudo original apontou consistência interna satisfatória $(\alpha=0,93)$. Para responder às questões, a criança deve selecionar uma das cinco opções de frequência: (1) nem um pouco, (2) um pouco, (3) mais ou menos, (4) bastante, (5) muitíssimo. Assim como para a escala YLOT e para a escala de Afetos Positivos e Negativos, essa escala foi adaptada para a utilização em crianças menores de oito anos. A aplicação foi individual e os itens foram lidos para a criança. Para auxiliar nas respostas, foi criada uma escala pictórica de cinco pontos, semelhante a da YLOT.

Tarefas Preditoras de Otimismo em Crianças - TAPOC (Bandeira, Giacomoni, \& Hutz, 2014b). A TAPOC é um instrumento que avalia o otimismo em crianças pequenas (quatro a oito anos) por meio de 12 historietas com desenhos coloridos em cartões de 20x15 centímetros. Cada item/cenário conta com três cartões desenhados, tal que o primeiro cartão apresenta uma cena inicial do enredo e os outros dois apresentam possíveis desfechos, um otimista e outro pessimista. É 
utilizada uma escala de respostas do tipo Likert de cinco pontos que varia de 1 a 5 , sendo que 1 corresponde à resposta "pode acontecer" e 5, "certamente vai acontecer". A escolha pessimista gera um resultado negativo e a escolha otimista um resultado positivo. Os resultados variam de $-5 \mathrm{a}+5$, sendo que os resultados mais altos indicam maior otimismo. $\mathrm{O}$ estudo original indicou consistência interna satisfatória $(\alpha=0,79)$.

Os instrumentos utilizados para avaliar o bem-estar subjetivo e otimismo dos pais estão descritos a seguir.

Escala de Afeto Positivo e Negativo - PANAS (Giacomoni \& Hutz, 1997). Essa escala é formada por dois fatores ortogonais, afeto positivo $(\alpha=0,88)$ e afeto negativo $(\alpha=0,86)$. Cada fator é composto por 20 adjetivos que representam humores e emoções dos sujeitos, como, por exemplo, amável, cuidadoso, aflito, impaciente. A frequência de ocorrência dos afetos é julgada pelos participantes numa escala de cinco pontos, quanto maior a ocorrência maior a pontuação na escala.

Escala de Satisfação de Vida (Giacomoni \& Hutz, 1997). Essa escala é composta por cinco itens que avaliam, de forma global, os aspectos cognitivos do bem-estar subjetivo. Exemplos de itens são: "A minha vida está próxima do meu ideal" e "Até agora eu tenho conseguido as coisas importantes que eu quero na vida”. Essa escala apresenta consistência interna adequada $(\alpha=0,91)$. A chave de respostas é uma escala Likert de sete pontos.

Revised Life Orientation Test - LOT-R (Bastianello \& Pacico, 2014). A LOT-R é uma escala construída para medir o otimismo disposicional, sendo uma versão reduzida e revisada do Life Orientation Test - LOT (Scheier \& Carver, 1985). O teste consiste em 10 itens, sendo três afirmativas sobre otimismo, três sobre pessimismo e quatro itens distratores cujos escores não são computados. Os participantes respondem às afirmativas indicando seu grau de concordância em uma escala Likert de cinco pontos, variando de "discordo plenamente" até "concordo plenamente". Os escores negativos do teste precisam ser invertidos para a análise estatística dos dados, com a finalidade de que os valores próximos a cinco sempre indiquem um maior grau de expectativa otimista da pessoa. A LOT-R apresenta adequada consistência interna $(\alpha=0,82)$.

Parent-rated Life Orientation Test of children - PLOT (Bandeira, Giacomoni, \& Hutz, 2014c). Essa medida utiliza o relato dos pais em relação ao otimismo dos filhos, o que facilita a investigação do otimismo em crianças pequenas. É composta por oito itens, elaborados a partir da LOT e da YLOT, além da criação de outros sobre a percepção dos pais quanto ao otimismo e o pessimismo de seus filhos. É respondida por meio de uma escala de respostas do tipo Likert de quatro pontos. Os estudos de adaptação e validação para a cultura brasileira apontaram consistência interna adequada para as mães $(\alpha=0,80)$ e para os pais $(\alpha=0,77)$.

\section{Procedimentos}

O contato com as escolas ocorreu por meio de visitas e reuniões com a equipe de direção e coordenação. Foi solicitada às escolas a concordância quanto à realização do estudo. Os pais ou responsáveis pelas crianças foram contatados por meio de um documento escrito, com esclarecimentos sobre a pesquisa, além da solicitação de assinatura no Termo de Consentimento Livre e Esclarecido. Salvaguardou-se a todos os participantes o direito de sigilo, voluntariado e interrupção da participação. O presente estudo está de acordo com a Resolução n ${ }^{\circ}$ 466/2012 do Conselho Nacional de Saúde, que versa sobre os aspectos éticos da pesquisa com seres humanos. Todos os procedimentos atenderam as recomendações do Comitê de Ética e Pesquisa do Instituto de Psicologia da UFRGS o qual aprovou o estudo sob o protocolo $\mathrm{n}^{\mathrm{O}} 22623$.

A coleta de dados das crianças foi realizada nas dependências das escolas, em salas de aula destinadas à realização da pesquisa no mesmo turno das aulas. Os instrumentos foram aplicados pela pesquisadora e por alunos graduandos de Psicologia previamente treinados. As crianças menores de nove anos responderam individualmente, enquanto as demais responderam de forma coletiva. Todos os alunos receberam informações sobre os objetivos do estudo por meio de uma linguagem apropriada para cada faixa etária. Foi dito aos participantes que gostaríamos de saber o que pensam a respeito de algumas coisas que fazem parte de suas vidas, e de como se sentem a respeito. Todos foram informados verbalmente que sua participação era voluntária e que poderiam interrompê-la a qualquer momento, sem nenhuma penalidade. Foram prestadas informações quanto ao sigilo e a confidencialidade dos dados coletados. Os participantes foram encorajados a responder honestamente e informados que não existiam respostas certas ou erradas. A coleta de dados foi realizada em uma única sessão com tempo aproximado de 45 minutos.

Os responsáveis pelas crianças receberam um envelope com os instrumentos a serem preenchidos por eles próprios. O envelope continha, além dos 
instrumentos direcionados aos pais e as mães, informações sobre a pesquisa e instruções de como proceder no preenchimento e devolução. Os dados dos pais e dos filhos foram relacionados por meio de uma numeração que substituiu os nomes presentes no termo de consentimento informado para salvaguardar o sigilo dos dados.

\section{Resultados}

A fim de caracterizar os níveis de otimismo e BES de pais e filhos, inicialmente, calcularam-se médias e desvios-padrão dos construtos acessados neste estudo. $\mathrm{Na}$ Tabela 1 são apresentadas as médias e desvios-padrão para cada escala por sexo e para crianças e adultos. As médias de meninos e meninas e homens e mulheres foram comparadas por meio do teste $t$ de Student. Entre meninos e meninas, verificaram-se diferenças significativas na subescala de pessimismo, sendo que os meninos apresentam média significativamente mais alta que as meninas, $t(360)=2,09 ; p=0,04 ; d=$ 0,22 . Em relação aos pais e mães, foi encontrada diferença significativa na satisfação de vida, sendo que as mães apresentaram médias significativamente superiores a dos pais, $t(120)=2,24 ; p=0,03 ; d=0,20$.

Para avaliar as relações de otimismo, afeto positivo, afeto negativo e satisfação de vida entre pais e filhos, foram calculadas correlações de Pearson. Os coeficientes de correlação podem ser visualizados na Tabela 2 . Destaca-se a correlação positiva entre pais e mães na percepção de otimismo dos seus filhos (medido pela
PLOT). Também se salientam as correlações positivas entre o otimismo dos filhos (TAPOC) e o afeto positivo das mães, e entre a satisfação de vida dos filhos e o otimismo das mães. Entre pais e mães destaca-se a correlação positiva para a satisfação de vida.

\section{Discussão}

Este estudo teve como objetivo verificar as relações de otimismo e BES entre pais e filhos. Os resultados corroboram achados de outras pesquisas que não encontraram relação entre o otimismo dos pais e otimismo dos filhos e entre o BES dos pais e o BES dos filhos (Hasan \& Power, 2002; Heinonen et al., 2005). Embora a literatura aponte para alguns fatores relacionados ao desenvolvimento do otimismo e do BES, como um ambiente familiar positivo (Ey et a., 2005; Heinonen et al., 2005) e pais autoritativos (Weber et al., 2003), é possível que tanto o otimismo quanto o BES dos filhos não seja um reflexo dos pais.

As correlações entre pais e filhos foram evidenciadas entre o otimismo dos filhos, nos resultados encontrados por meio da TAPOC, e os afetos positivos das mães. Outra correlação encontrada foi entre a satisfação de vida dos filhos e o otimismo das mães. Mães que experienciam maiores níveis de afetos positivos têm filhos mais otimistas e mães mais otimistas têm filhos mais satisfeitos com a vida. Não foram encontradas correlações entre otimismo e BES dos pais com os filhos. A satisfação de vida materna tem sido relacionada a aspectos positivos do desenvolvimento infantil,

Tabela 1

Médias de Pais e Filhos para Bem-Estar Subjetivo e Otimismo

\begin{tabular}{|c|c|c|c|c|c|c|c|}
\hline & $\begin{array}{c}\text { Crianças-Todos } \\
M(D P) \\
\end{array}$ & $\begin{array}{c}\text { Meninos } \\
M(D P)\end{array}$ & $\begin{array}{l}\text { Meninas } \\
M(D P)\end{array}$ & $d$ & $\begin{array}{c}\text { Pais } \\
M(D P)\end{array}$ & $\begin{array}{c}\text { Mães } \\
M(D P)\end{array}$ & $d$ \\
\hline ТАРOC & 4,01 (1,23) & $3,94(1,31)$ & $4,08(1,16)$ & 0,12 & - & - & - \\
\hline YLOT total & $3,27(0,51)$ & $3,23(0,54)$ & $3,30(0,48)$ & 0,13 & - & - & - \\
\hline YLOT otimismo & $3,42(0,52)$ & $3,42(0,54)$ & $3,42(0,51)$ & 0,01 & - & - & - \\
\hline YLOT pessimismo & $1,89(0,66)$ & $1,97(0,69)$ & $1,82(0,62)$ & $0,22^{*}$ & - & - & - \\
\hline Afetos positivos & $4,33(0,46)$ & $4,19(0,62)$ & $4,22(0,58)$ & 0,06 & $3,37(0,55)$ & $3,30(0,66)$ & 0,12 \\
\hline Afetos negativos & $1,86(0,60)$ & $1,89(0,81)$ & $1,83(0,76)$ & 0,08 & $2,03(0,58)$ & $2,13(0,65)$ & 0,16 \\
\hline Satisfação de vida & $4,21(0,78)$ & $4,30(0,48)$ & $4,36(0,43)$ & 0,14 & $5,08(1,15)$ & $5,32(1,20)$ & $0,20^{*}$ \\
\hline PLOT & - & - & - & - & $3,25(0,54)$ & $3,18(0,62)$ & 0,11 \\
\hline LOT-R & - & - & - & - & $4,15(0,67)$ & $4,26(0,62)$ & 0,18 \\
\hline
\end{tabular}

Nota. * Verificaram-se diferenças significativas de médias entre sexo, $p<0,05$. TAPOC - Tarefas Preditoras de Otimismo em Crianças; YLOT - Youth Life Orientation Test, PLOT - Parent-rated Life Orientation Test of children; LOT-R - Life Orientation Test Revised. 


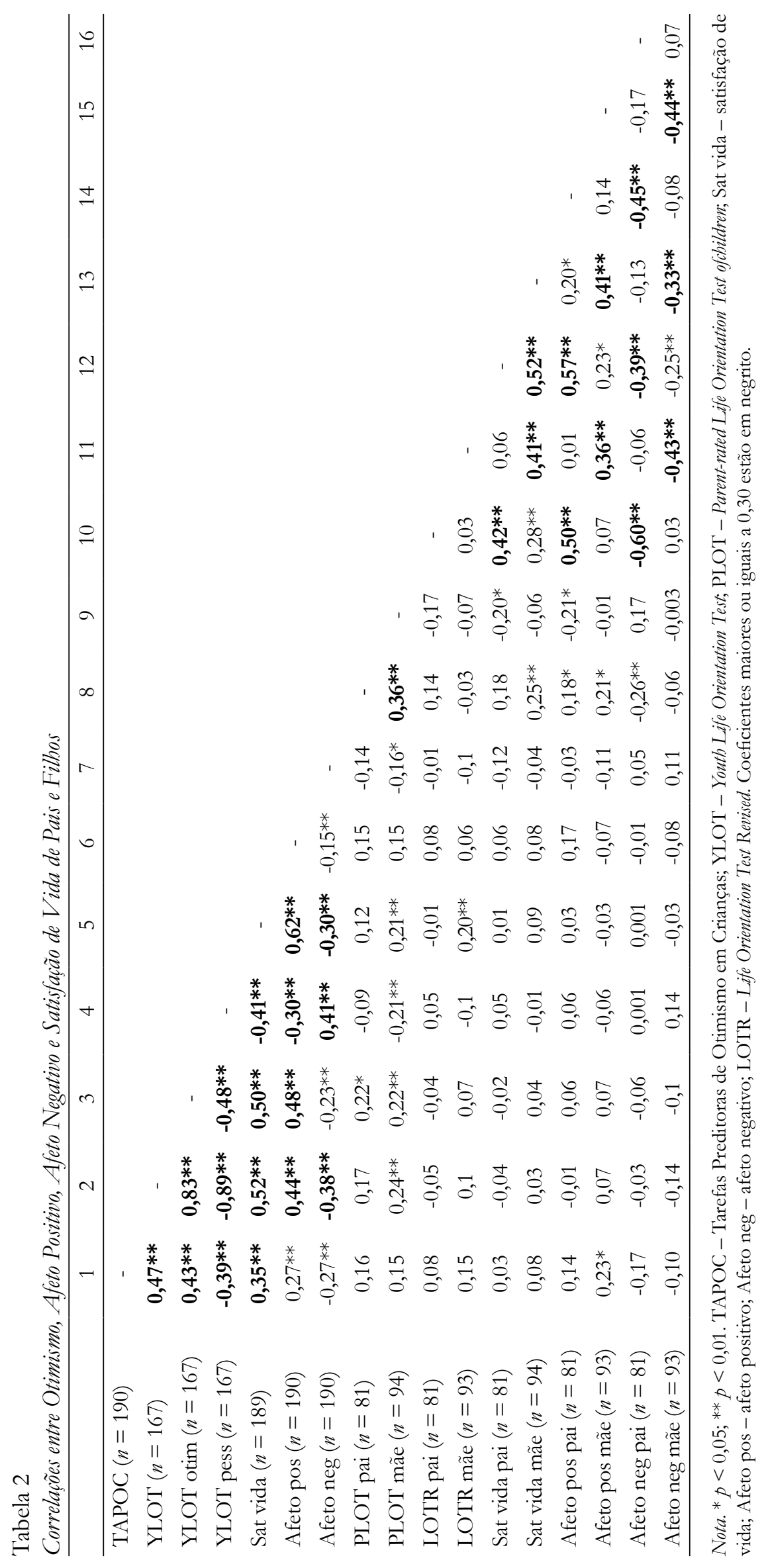


como a diminuição nos problemas de comportamento dos filhos e aumento do desempenho cognitivo das crianças (Berger \& Spiess, 2011). Os resultados do estudo de Berger e Spiess (2011) indicaram que quanto mais satisfeita a mãe está com sua vida, melhor a habilidade verbal da criança e menores seus níveis de problemas socioemocionais.

Os dados desse estudo sugerem que quanto mais satisfeita e feliz a mãe está, melhor é a sua capacidade de desempenhar o papel da maternagem. Quando as mães apresentam humor positivo, elas tendem a interagir verbalmente e fazer afirmações positivas junto a seus filhos. Da mesma forma, elas interagem mais com seus filhos, são mais responsivas e mais próximas e eles (Desjardins et a., 2008). Variáveis maternas têm sido relacionadas tanto a indicadores de saúde quanto de doença entre os filhos (Desjardins et al., 2008; Heinonen et al., 2005; Lemola et al., 2010), como indica os resultados de um estudo no qual o pessimismo das mães correlacionou com o pessimismo dos filhos, enquanto o otimismo não apresentou correlação (Hasan \& Power, 2002). Quando a mãe não está satisfeita com sua vida é mais provável que sua atenção fique concentrada em seus próprios problemas e dificuldades, e não tanto nas necessidades da criança (Desjardins et al., 2008).

Considerando que os resultados deste estudo identificaram correlações positivas entre a satisfação de vida das mães e a satisfação de vida dos pais, é possível concluir que pais e mães mais satisfeitos permitem o desenvolvimento de características positivas em seus filhos, como o otimismo e o bem-estar. A parentalidade influencia o desenvolvimento infantil repercutindo nas competências sociais e cognitivas da criança (Desjardins et al., 2008). É provável que maiores níveis de BES e otimismo estejam relacionados ao comportamento parental que influencia o desenvolvimento positivo. É possível afirmar que pais otimistas apresentam expectativas mais positivas e mais realistas em relação aos filhos, uma vez que apresentam expectativas positivas de forma generalizada. Assim, é possível que se sintam mais competentes ao exercer a parentalidade, estimulem mais seus filhos e invistam mais na sua educação. Da mesma forma, pode-se admitir que a postura desses pais seja mais proativa, fazendo com que eles demonstrem mais confiança e sejam mais persistentes em suas tarefas como pais.

De maneira geral, as médias de otimismo e BES encontradas neste estudo não apresentaram diferenças significativas entre os sexos. Em relação ao BES, meninas e meninos apresentaram níveis semelhantes.
A literatura sobre satisfação de vida em crianças sustenta esses achados ao afirmar que variáveis, como sexo, idade, ocupação dos pais ou inteligência contam menos para a satisfação de vida das crianças do que, por exemplo, as relações interpessoais (Gilman \& Huebner, 2003; Park, 2004). Da mesma forma, em relação ao otimismo, meninas e meninos apresentaram resultados semelhantes. As diferenças foram encontradas nos níveis de pessimismo, onde os meninos apresentaram médias mais altas do que as meninas. Esses achados corroboram estudos que identificaram maiores níveis de pessimismo entre os meninos (Ey et al., 2005; Lemola et al., 2010).

Entretanto, tais resultados apoiam as divergências quanto à dimensionalidade do construto. Por um lado, o modelo bidimensional sustenta o otimismo e o pessimismo como fatores distintos, enquanto o modelo unidimensional sugere a existência de dois polos opostos do mesmo contínuo (Carver et al., 2010; Ey et al., 2005; Scheier \& Carver, 1985; Scheier et al., 1994). A validade e a utilidade desses modelos devem ser estimadas com base na sua utilidade prática. Por isso, mais estudos são necessários para avaliar com maior propriedade essas questões.

Em relação aos pais e mães, as mães apresentaram médias significativamente superiores aos pais no que diz respeito à satisfação de vida. Esses resultados diferem dos achados do estudo desenvolvido por Tomasi (2013), o qual indicou níveis menores de satisfação de vida entre as mulheres adultas e adolescentes. Estudos desenvolvidos com crianças (Giacomoni \& Hutz, 2008; Gilman \& Huebner, 2003; Huebner, 1991) não apontaram para diferenças entre os sexos em relação a essa variável. Também foram identificadas correlações positivas entre a satisfação de vida das mães e a satisfação de vida dos pais.

Diferente do esperado inicialmente, não foram encontradas correlações entre o otimismo dos pais e otimismo dos filhos ou entre o BES dos pais e o BES dos filhos. Entretanto, verificou-se que variáveis maternas, como afetos positivos e otimismo, estão relacionadas ao otimismo e a satisfação de vida dos filhos, respectivamente. Cabe salientar que os resultados desse estudo não permitem generalização para a população em geral. Mais estudos são necessários para um melhor entendimento da complexidade das relações entre pais e filhos. Para futuras investigações, sugere-se avaliar a relação do otimismo e do BES em crianças e entre pais e filhos considerando-se o período da adolescência. $\mathrm{O}$ conhecimento mais amplo dessas relações pode auxiliar 
na compreensão dos diferentes fatores que compõem as relações familiares e assim promover de forma proativa o desenvolvimento positivo.

\section{Referências}

Bandeira, C. M., Giacomoni, C. H., \& Hutz, C. S. (2014a). Estudos de adaptação e validação brasileira do Youth Life Orientation Test (YLOT). Manuscrito em preparação.

Bandeira, C. M., Giacomoni, C. H., \& Hutz, C. S. (2014b). Estudos de adaptação e validação brasileira do Parent-rated Life Orientation Test of children (PLOT). Manuscrito em preparação.

Bandeira, C. M., Giacomoni, C. H., \& Hutz, C. S. (2014c). Tarefas Preditoras de Otimismo em Crianças (TAPOC) - Construção e evidências de validade. Manuscrito em preparação.

Bastianello, M. R., \& Pacico, J. C. (2014). Otimismo. Em C. S. Hutz (Ed.), Avaliação em psicologia positiva (pp. 49-55). Artmed: Porto Alegre, Brasil.

Berger, E. M., \& Spiess, K. K. (2011). Maternal life satisfaction and child outcomes: Are they related? Journal of Economic Psychology, 32, 142-58.

Carver, C. S., Scheier, M. F., \& Segerstrom, S. C. (2010). Optimism. Clinical Psychology Review, 30, 879-889.

Chang, E. C. (2002). Optimism-pessimism and stress appraisal: Testing a cognitive interactive model of psychological adjustment in adults. Cognitive Therapy and Research, 26, 675-690.

Desjardins, J., Zelenski, J. M., \& Coplan, J. R. (2008). An investigation of maternal personality, parenting styles, and subjective well-being. Personality and Individual Differences, 44, 587-597.

Diener, E. (2000). Subjective well-being: The science of happiness and a proposal for a national index. American Psychologist, 55, 34-43.

Diener, E., Oishi, S., \& Lucas, R. E. (2003). Personality, culture, and subjective well-being: Emotional and cognitive evaluations of life. Annual Review of Psychology, 54, 403-425.

Ey, S., Hadley, W., Allen, D. N., Palmer, S., Klosky, J., Deptula, D., Thomas, J., \& Cohen, R. (2005). A new measure of children's optimism and pessimism: The youth life orientation test. Journal of Child Psychology and Psychiatry, 46(5), 548-558.
Giacomoni, C. H., Bandeira, C. M., \& Zanon, C. (2014). Escala multidimensional de satisfação de vida para crianças-versão redu₹ida. Manuscrito em preparação.

Giacomoni, C. H., \& Hutz, C. S. (2006). Escala de afeto positivo e negativo para crianças: Estudos de construção e validação. Revista Semestral da Associação Brasileira de Psicologia Escolar e Educacional, 10(2), 235-245.

Giacomoni, C. H., \& Hutz, C. S. (1997). A mensuração do bem-estar subjetivo: Escala de afeto positivo e negativo e escala de satisfação de vida [Resumos]. Em Sociedade Interamericana de Psicologia (Org.), Anais XXVI Congresso Interamericano de Psicologia (p. 313). São Paulo, SP: SIP.

Giacomoni, C. H., \& Hutz, C. S. (2008). Escala multidimensional de satisfação de vida para crianças: Estudos de construção e validação. Estudos de Psicologia, 25(1), 23-35.

Gilman, R., \& Huebner, E. S. (2003). A review of life satisfaction research with children and adolescents. School Psychology Quarterly, 18, 192-205.

Hasan, N., \& Power, T. G. (2002). Optimism and pessimism in children: A study of parenting correlates. International Journal of Behavioral Development, 26(2), 185-191.

Heinonen, K., Räikkönen, K., \& Keltikangas-Järvinen, L. (2005). Dispositional optimism: Development over 21 years from the perspectives of perceived temperament and mothering. Personality and Individual Differences, 38, 425-435.

Huebner, E. S. (1991). Correlates of life satisfaction in children. School Psychology Quarterly, 6, 103-111.

Larson, R. (2006). Positive youth development, willful adolescents, and mentoring. Journal of Community Psychology, 34(6), 677-689.

Lemola, S., Räikkönen, K., Matthews, K. A., Scheier, M. F., Heinonen, K., Pesonen, A.-K., Komsi, N., \& Lahti, J. (2010).A new measure for dispositional optimism and pessimism in young children. European Journal of Personality, 24, 71-84.

Lerner, R. M., Almerigi, J. B., Theokas, C., \& Lerner, J. V. (2005). Positive youth development, a view of the issues. The Journal of Early Adolescence, 25(1), 10-16. 
Park, N. (2004). The role of subjective well-being in positive youth development. The ANNALS of the American Academy of Political and Social Science, 591, 25-39.

Roberts, M.C., Brown, K.J., Johnson, R.J., \& Reinke J. (2005). Positive Psychology in children: Development, prevention, and promotion. In S. J. Lopez \& C. R. Snyder (Eds.), Handbook of Positive Psychology (pp. 663-675). New York: Oxford University Press.

Scheier, M. F., Carver, C. S., \& Bridges, M. W. (1994). Distinguishing optimism from neuroticism (and trait anxiety, self-mastery, and self-esteem): A reevaluation of the Life Orientation Test. Journal of Personality and Social Psychology, 67, 1063-1078.

Scheier, M. F., \& Carver, C.S. (1985). Optimism, coping, and health. Assessment and implications of generalized outcome expectancies. Health Psychology, 4, 219-247.
Seligman, M. E. P., \& Csikszentmihalyi, M. (2000). Positive psychology: An introduction. American Psychologist, 55, 5-14.

Tomasi, L. M. B. (2013). Relação entre o bem-estar subjetivo de pais e filhos adolescentes a partir de diferentes medidas de avaliação (Tese não publicada). Programa de Pós-Graduação em Psicologia do Desenvolvimento, Instituto de Psicologia, Universidade Federal do Rio Grande do Sul, Porto Alegre, Brasil.

Weber, L. N. D., Brandenburg, O. J., \& Viezzer, A. P. (2003). A relação entre o estilo parental e o otimismo da criança. Psico-USF, 8(1), 71-79.

Recebido: 08/07/2014

$1^{a}$ reformulação: $24 / 10 / 2014$

$2^{\mathrm{a}}$ reformulação: $13 / 12 / 2014$

Aprovado: 05/02/2015

Sobre os autores:

Cláudia de Moraes Bandeira é professora do departamento de Psicologia da UNILASALLE Canoas, RS e doutora em Psicologia pelo programa de pós-graduação da Universidade Federal do Rio Grande do Sul. Áreas de interesse em pesquisa: Avaliação Psicológica, Psicologia Positiva, Psicologia do Desenvolvimento Infantil e da Adolescência, Família e Bullying.

Jean Carlos Natividade é professor do programa de pós-graduação em Psicologia Clínica da Pontifícia Universidade Católica do Rio de Janeiro e doutor em Psicologia pelo programa de pós-graduação em Psicologia da Universidade Federal do Rio Grande do Sul. Áreas de interesse: diferenças individuais e avaliação da personalidade.

Claudia Hofheinz Giacomoni é professora do departamento de Psicologia do Desenvolvimento e da Personalidade, programa de pós-graduação em Psicologia, Instituto de Psicologia da UFRGS e doutora em Psicologia UFRGS. Áreas de interesse em pesquisa: Avaliação Psicológica, Psicologia Positiva e Psicologia do Desenvolvimento Infantil e da Adolescência.

Contato com os autores:

Universidade Federal do Rio Grande do Sul

Rua Ramiro Barcelos, 2600, sala 101, Porto Alegre - RS

CEP: 900035-003

E-mail:kkbandeira@hotmail.com

Psico-USF, Bragança Paulista, v. 20, n. 2, p. 249-257, mai./ago. 2015 